\title{
The legacy of coronary sinus interventions: Endogenous cardioprotection and regeneration beyond stem cell research
}

Werner Mohl, MD, PhD, ${ }^{a}$ Stefan Mina, BME, ${ }^{\mathrm{b}}$ Dejan Milasinovic, ${ }^{\mathrm{a}}$ Hirofumi Kasahara, MD, PhD, ${ }^{\text {a }}$ and Sheng Wei, $\mathrm{MD}^{\mathrm{a}}$

From the Departments of Cardiothoracic Surgery, Medical University of Vienna, Vienna, Austria, a and Biomedical Engineering, Austrian Research Centers, Wiener Neustadt, Austria. ${ }^{\text {b }}$

Received for publication March 3, 2008; revisions received April 18, 2008; accepted for publication May 15, 2008.

Address for reprints: Werner Mohl, MD, PhD, Department of Cardiothoracic Surgery, University of Vienna, Waehringerguertel 18-20, 1090 Vienna, Austria (E-mail: werner.mohl@meduniwien.ac.at).

J Thorac Cardiovasc Surg 2008;136:1131-5 0022-5223/\$34.00

Copyright (C) 2008 by The American Association for Thoracic Surgery

doi:10.1016/j.jtcvs.2008.05.034
Even when laws have been written down, they ought not always to remain unaltered.

\author{
-Aristotle, Politics \\ Greek critic, philosopher, physicist, and zoologist (384 BC-322 BC)
}

\section{The Road to Reality}

$\mathrm{G}$ aining new ground in scientific comprehension needs to radically overrule current beliefs reversing constraints of popular science genre with a unique combination of respect for the complexity of nature, the achievements of the past, and striving toward the final goal to fight disease.

The coronary sinus, as minimally invasive access to the diseased heart, is an attractive route occupying generations of researchers. As in many other scientific developments and achievements in biology and medicine, coronary sinus interventions lack uniformity and leave observers with plenty of uncertainty. Facts and myths are scattered, leaving ground for speculations and piling up constraints for the future. These constraints include the potential to recapitulate old mistakes or ill-fated avenues but also leave room to bridge to new ideas on a sound basis of new understanding of mistaken and unappreciated information.

The recent reports on the development of a coronary sinus reducer stent for the treatment of chronic refractory angina pectoris have focused new interest on the concept of coronary sinus interventions necessitating a second look at widely established knowledge and a redirection of a potential "fausse route" jeopardizing scientific targets. Permanent elevation of right-sided pressures in the heart is known to occur during the development of cardiac failure. Our clinical predecessors knew that patients had a lucid interval with improvement of symptoms as soon as right atrial pressure transmitted into the cardiac venous system, a phenomenon that is known also from the Fontan circulation. ${ }^{1}$

Although this improvement does not last very long but rather deteriorates promptly, it is certainly the basis for treatment options like the coronary reducer stent.

\section{The Past and the Present of Coronary Sinus Interventions}

The coronary sinus has been a surgical domain from its very beginning, culminating in the methodology of retrograde cardioplegia as a powerful means to protect the heart during global ischemia. Besides the current standard use of pacing electrodes for biventricular pacemakers or implantable cardioverter-defibrillators, various techniques involving alterations of blood flow and pressure in coronary veins have been and still are used in clinical practice and scientific research. Although some of them never reached widespread clinical attention, the common goal of these methods is the enhancement of retrograde perfusion to deprived myocardium, protection during ischemia, and the prevention of reperfusion injury.

After the first clinical application of the Beck II procedure, a second "tidal wave" of interest arose at the beginning of the interventional era. However, most of the concepts have been abandoned because of the lack of a plausible explanation of the underlying mechanisms as well as the advancement of coronary artery interventions. ${ }^{2}$ 
The development of percutaneous coronary interventions and stenting in acute coronary syndromes clearly shifted the interest away from the coronary venous system. Despite considerable improvements in experimental and early clinical studies, the existence of two schools of thought and the unilaterally aligned interest of the medical device industry drowned an otherwise interesting and clinically feasible concept for almost three decades. ${ }^{3}$ Interestingly enough, the current revival of interest is based on stem cell research, molecular science, and the potential of regeneration on the one hand and the clinical interest on advancement in interventional technology on the other. The principles of the Beck II procedure, which was assumed to be obsolete with the introduction of coronary artery bypass grafting, gave many investigators early knowledge on the principle of how to supply deprived myocardium with oxygenated blood retroperfused in the presence of coronary artery obstruction. According to Paz, ${ }^{4}$ this was also the motivation to develop the reducer stent (letter to the editor). What was less appreciated is that there are historic reports showing that new vessels are formed as a consequence of the Beck II operation. ${ }^{2,5}$ One very important and successful survivor of these techniques remains the application of retrograde cardioplegia, which helped to protect the myocardium even in an era of changing surgical demographics and dealing with more difficult and complex cases every day.

Three decades ago the advances in catheter technology led to the development of a more sophisticated method of synchronizing retroperfusion (SRP) to diastole, allowing normal drainage during systole aiming to penetrate the venous system more effectively. The method was even evaluated as support of high-risk angioplasty ${ }^{6}$ and in patients with acute coronary syndromes, but the concept had to be abandoned due to the lack of efficiency and missed clinical end points. A further advancement of the SRP concept was selective synchronized suction and retroperfusion (SSR). SSR relies on a selective access of the local coronary vein draining the ischemic area. In contrast to SRP, the regional cardiac veins are emptied by suction of blood before retroinfusion.

\section{The Role of Pressure Elevation in the Coronary Venous Circulation}

A different philosophy is the application of pressure-controlled intermittent coronary sinus occlusion (PICSO). PICSO works without additional retroinfusion of arterial blood. The developed coronary sinus occlusion pressure is a consequence of the squeezing action of the heart during systole and coronary venous flow. This allows a pressure wave front to enter the ischemic coronary bed, redistributing blood from normally perfused veins retrogradely into the microcirculation of deprived myocardium. After the systolic pressure reaches a plateau, the vascular capacitance is completely filled and the occlusion is released, allowing sufficient drainage. The significant difference compared with SRP and SSR is that the optimal occlusion time lasts over several heartbeats and varies depending on myocardial function and myocardial perfusion. The occlusion and release times are derived from the coronary sinus pressure, which is measured through the inner lumen of the dedicated balloon catheter. Early clinical evaluations have shown benefits of PICSO during coronary artery bypass grafting. ${ }^{7}$ and experimental studies demonstrate the salvage potential for the ischemic myocardium. ${ }^{8}$

\section{The Paradigm Change of Oxygen Delivery Toward Endogenous Cardioprotection and Regeneration}

Originally, all methods involving a retrograde myocardial supply have been thought to act only by redistribution of blood flow, especially emphasizing the oxygenated component of the reperfusate. To reverse myocardial blood flow totally results in normal cardiac action, at least in an empty beating and vented heart, as can be observed during aortic crossclamping and after the reversal of cardioplegic arrest with warm blood. In regional ischemia, however, the impedance of antegrade flow prevents effective nutritive backflow of oxygenated blood, reversing venous flow toward underperfused areas.

Although experience should have led toward a different school of thought, it is only recently that a clear picture of coronary sinus interventions unfolds, bringing endogenous molecular pathways into focus.

\section{Relating Coronary Sinus Interventions and Mechanotransduction}

Recently, mechanotransduction and activation of venous endothelium by pulsatile stretch of the elevated coronary venous pressure have been suggested to be the initial impulse for the improvements observed with coronary sinus interventions. The quantity of this mechanical stimulus is directly related to the effectiveness of the method. In contrast to intermittent occlusion techniques fully utilizing this principle, the coronary sinus reducer stent causes a permanent but small increase in the coronary venous pressure and results in diminutive endothelial shear stress.

A permanent reduction of coronary sinus flow worsens coronary perfusion, including reflexes such as bradycardia and hypotension. The instance in which this was tolerated in a number of patients having the Beck II procedure is based on the reduced coronary inflow, the patchy microcirculatory perfusion of the diseased heart, and in this respect Paz is right in stating," If we cannot increase the coronary arterial inflow into deprived myocardium let's limit the coronary venous outflow."

As seen in several scientific evaluations of our own group, optimizing the timing of venous pressure elevation defines the shifted blood volumes and helps to control unwanted side effects. ${ }^{9}$ 


\section{The Pluripotency of Coronary Venous Endothelium}

Combining reports on the effects of mechanotransduction with the hypothesis of the resurrection of inborn pathways used during organogenesis for the repair of an adult failing heart (embryonic recall) meets the claim of clinical significance and a more consistent understanding of coronary sinus interventions. $^{10,11}$

The central contribution in this hypothesis is the discovery of Zheng and associates, ${ }^{12}$ who demonstrated that coronary microvascular endothelial cells under shear stress upregulate vascular endothelial growth factor (VEGF). This mechanical kinetic action of blood flow is also modeling the developing heart. $^{13}$

Pulsatile stretch on coronary venous endothelium by intermittent elevated pressure resumes actions in the developing heart and opens dormant pathways in the adult heart.

This "embryonic recall" might therefore enable revascularization and structural regeneration, closing the cycle to the histologic observations of the Beck II procedure and the observations in PICSO experiments relating VEGF upregulation and coronary venous pressure elevation. ${ }^{6}$ The same relationship was found by Syeda and associates ${ }^{7}$ between myocardial salvage and developed coronary sinus pressure in experimental infarction in different species. In this case, a high pressure is assumed to cause significant cyclic shear stress and pulsatile stretch of the endothelium. VEGF and other growth factors, such as the group of fibroblast growth factors, are keys to angiogenesis and vasculogenesis. Grunewald and colleagues ${ }^{14}$ found that VEGF induces perivascular expression of the chemokine SDF1, homing circulating progenitor cells and enhancing in situ proliferation of resident activated endothelial cells, ${ }^{14}$ thus promising that angiogenesis and regeneration can be achieved by mobilization, attraction, and implantation of pluripotent cells.

Vasodilatation of the myocardial vasculature is an important target in preventing damage in deprived myocardial zones. It appears to be essential in the salvage of ischemic border zones and subsequently significant infarct size reduction. The similarity of myocardial salvage of all coronary sinus interventions, including retroperfusion of oxygenated blood, underscores that vasodilatation and enhancement of collateral flow, especially in border zones, is the pathophysiologic substrate in cardioprotection. Activation of obvious pluripotent venous endothelium and the depending molecular cascade may also play a hitherto unappreciated role in retrograde cardioplegia and warrants further studies.

\section{Toward a New Understanding}

Under the perspective mentioned earlier, a clear pattern unfolds: with all coronary sinus interventions, a periodic pulsatile stretch, activating venous endothelium, seems to be the starting point of observed effects; the amplitude of the pressure peaks in direct relation to optimal effectiveness. The semantic differences in retroperfusion concepts versus intermittent occlusion are therefore irrelevant inasmuch as the retroperfused blood cushion also periodically increases venous outflow impedance followed by shear stress and pulsatile stretch of endothelium during drainage in the coronary venous system. Positive effects might have been obscured by a limited amount of retroperfused volume and a less than optimal pressure increase and therefore less than optimal activation of venous endothelium. This inconsistency of retroperfusion, however, seems to be solved by the optimized action of PICSO.

Still, there is a great deal of ambivalence regarding the wanted positive effects of high venous pressure on the activation of venous endothelium and the influences on coronary perfusion, thus requiring an optimization as it is achieved with PICSO scaling physiologic responses individually and minimizing side effects.

Claiming clinical significance, we are now focusing on the activation of venous endothelium embedding coronary sinus interventions in the context of modern technology and the background of the clinical imperative.

Beneficial effects can also be expected in prevention of reperfusion injury, still a major threat in cardiac surgery. Most protective effects are associated with the release of nitric oxide, which is activated in the presence of shear stress on the endothelium. Therefore, it appears obvious to focus on therapeutic methods that can generate this kind of mechanical stress, such as PICSO, which provides an optimal activation of venous endothelium through pulsatile stretch and shear stress.

In Figure 1, the most important pathways resulting from an activation of venous endothelium leading to cytoprotection, redistribution of flow and washout, and finally toward myocardial salvage and regeneration are depicted.

A good example for the current paradigm change is also retrograde cardioplegia. Routinely each day, in hundreds of cardiac operations, we are using principles despite being totally unaware of their potential help during surgery (ie, vasodilatation of the coronary circulatory bed) and their implications in structural regeneration. If applied correctly and with optimal technology, we will gain another important tool in current clinical practice. It is quite certain that findings on the activation of coronary venous endothelium will influence how and when we use retrograde cardioplegia. Mechanotransduction of the retrograde flowing perfusate on venous endothelium and elevated pressure not only should result in vasodilatation of the coronary bed but also should change the pattern from reperfusion injury toward regeneration.

The long-lasting history of coronary sinus interventions showed a roller coaster-like wave form of research interest and clinical focus. The current unmet clinical need in myocardial regeneration eventually will bring these interventions back into the mainstream of interventional cardiology and myocardial protection during cardiac surgery. 


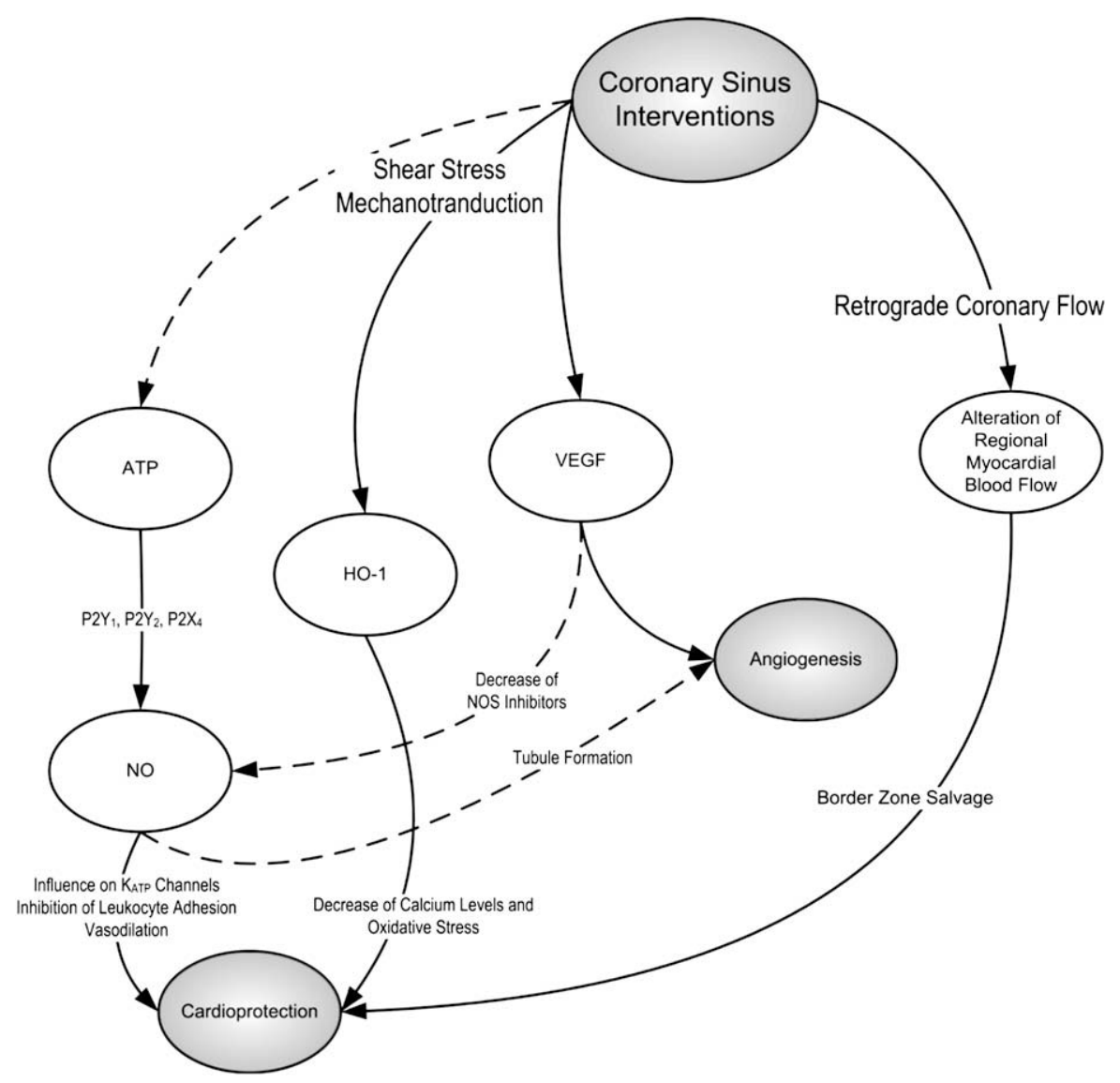

Figure 1. Mechanisms of coronary sinus interventions to angiogenesis, salvage, and cardioprotection. Mechanotransduction on the coronary vasculature induces vasodilatation and enhances angiogenesis in the ischemic myocardium. Enhancement of collateral flow in the border zone results in myocardial salvage. Biomechanical intervention of PICSO (since it resembles the optimal intervention for mechanotransduction) also potentially protects from reperfusion injury by inducing washout, thus protecting ischemic microcirculation. ATP, Adenosine triphosphate; VEGF, vascular endothelial growth factor; NO, nitric oxide; NOS, nitric oxide synthase; $\mathrm{HO}-1$, heme oxygenase-1.

Of special interest is the comparison between intra-aortic balloon pumping and PICSO reported by Lazar and associates, ${ }^{15}$ showing a synergistic effect of both methods on salvage and reports on the clinical potential of PICSO during myocardial infarction. The clinical potential of PICSO also extends as adjunct therapy in acute myocardial infarction in revascularization procedures by limiting myocardial necrosis and exceeding by far other clinically available methods. ${ }^{10}$

\section{Conclusions}

Periodic and physiologically timed pressure elevation within the coronary venous circulation, since it is based on the primary benefactor, namely activation of venous endothelium, is the legacy of coronary sinus interventions. To the best of our current knowledge, the therapeutic range is determined by a pressure control, taking into account the impedance changes within the coronary circulation. With today's existing technology, this method may lead to a revival of our perception on current myocardial protection via the coronary sinus in cardiosurgical as well as in the interventional clinical arena.

Especially in our surgical discipline, activation of coronary venous endothelium by periodic controlled elevation of the coronary sinus pressure and inducing shear stress by multiple doses of retrograde cardioplegia during global cardiac arrest seem to be of advantage for the future of cardiac surgery. Regenerative efforts in a quiescent organ are supporting natural laws of recovery otherwise far beyond any interventional domain.

\section{References}

1. Miura T, Hiramatsu T, Forbess JM, Mayer JE. Effects of elevated coronary sinus pressure on coronary blood flow and left ventricular function: implications after the Fontan operation. Circulation. 1995;92:298-303.

2. Beck CS, Stanton E, Batiuchok W, Leiter E. Revascularization of heart by graft of systemic artery into coronary sinus. JAMA. 1946;137:436-45.

3. Mohl W. The momentum of coronary sinus interventions clinically. Circulation. 1988;77:6-12.

4. Paz, et al. Coronary sinus reducer stent. J Thorac Cardiovasc Surg. 2008; 136:1390.

5. Weigel G, Kajgana I, Bergmeister H, Riedl G, Glogar HD, Gyöngyösi M, et al. Beck and back: a paradigm change in coronary sinus interventions - pulsatile stretch on intact coronary venous endothelium. J Thorac Cardiovasc Surg. 2007;133:1581-7.

6. Kar S, Drury JK, Hajduczki I, Eigler N, Wakida Y, Litvack F, Buchbinder N, Marcus H, Nordlander R, Corday E. (1991) Synchronized coronary venous retroperfusion for support and salvage of ischemic myocardium during elective and failed angioplasty. $\mathrm{J}$ Am Coll Cardiol. 1991;18:271-82.

7. Mohl W, Simon P, Neumann F, Schreiner W, Punzengruber C. Clinical evaluation of pressure-controlled intermittent coronary sinus occlusion: randomized trial during coronary artery surgery. Ann Thorac Surg. 1988;46:192-201. 
8. Syeda B, Schukro C, Heinze G, Modaressi K, Glogar D, Maurer G, et al. The salvage potential of coronary sinus interventions: meta-analysis and pathophysiologic consequences. J Thorac Cardiovasc Surg. 2004;127: 1703-12.

9. Mohl W, Kajgana I, Bergmeister H, Rattay F. Intermittent pressure elevation of the coronary venous system as a method to protect ischemic myocardium. 10.1510/icvts.2004.095364. Interact Cardiovasc Thorac Surg. 2005;4:66-9.

10. Mohl W, Komamura K, Kasahara H, Heinze G, Glogar D, Hirayama A, et al. Myocardial protection via the coronary sinus: long term effects of intermittent coronary sinus occlusion as adjunct to reperfusion in acute myocardial infarction. Circ J. 2008;72:526-33.

11. Mohl W. Embryonic recall: myocardial regeneration beyond stem cell transplantation. Wien Klin Wochenschr. 2007;119:333-6.
12. Zheng W, Seftor EA, Meininger CJ, Hendrix MJ, Tomanek RJ. Mechanisms of coronary angiogenesis in response to stretch: role of VEGF and TGF-beta. Am J Physiol Heart Circ Physiol. 2001;280: H909-17.

13. Groenendijk BCW, Hierck BP, Vrolijk J, Baiker M, Pourquie MJBM, Gittenberger-de Groot AV, et al. Changes in shear stress-related gene expression after experimentally altered venous return in the chicken embryo. Circ Res. 2005;96:1291-8.

14. Grunewald M, Avraham I, Dor Y, Bachar-Lustig E, Itin A, Jung S, et al. VEGF- induced adult neovascularization: recruitment, retention and role of accessory cells. Cell. 2006;124:175-89.

15. Lazar HL, Yang XM, Rivers S, Treanor P, Bernard S, Shemin RJ. Retroperfusion and balloon support to improve coronary revascularization. J Cardiovasc Surg. 1992;33:538-44. 